\title{
About the Infinite Repetition of Histories in Space *
}

\author{
Francisco José SOLER GIL and Manuel ALFONSECA
}

Received: 03.07.2013

Final version: 10.12 .2013

BIBLID [0495-4548 (2014) 29: 81; pp. 361-373]

DOI: $10.1387 /$ theoria. 9951

ABSTRACT: This paper analyzes two different proposals, one by Ellis and Brundrit, based on classical relativistic cosmology, the other by Garriga and Vilenkin, based on the DH interpretation of quantum mechanics. Both conclude that, in an infinite universe, planets and beings must be repeated an infinite number of times. We point to possible shortcomings in these arguments and conclude that the idea of an infinite repetition of histories in space cannot be considered, strictly speaking, a consequence of present physics and cosmology.

Keywords: infinite universe; classical cosmology; quantum physics.

RESUMEN: En este artículo se analizan dos propuestas diferentes: una de Ellis y Brundrit, basada en la cosmología relativista clásica, y la otra de Garriga y Vikenkin, basada en la interpretación DH de la mecánica cuántica. En ambas se concluye que, en un universo infinito, los planetas y los seres deberían repetirse un número infinito de veces. Indicaremos algunas posibles insuficiencias de estos argumentos y concluiremos que la idea de una repetición infinita de historias en el espacio no puede considerarse, en sentido estricto, una consecuencia de la física y la cosmología actuales.

Palabras clave: universo infinito; cosmología clásica; física cuántica.

\section{Introduction}

The notion that events, and series of events, repeat themselves infinitely, making history happen once and again, is very old. In the West, this idea is at least as old as philosophy and it can also be found in even older traditions of mythic thought (see Eliade 1954). ${ }^{1}$

These repetitions are usually presented as recurrences in time, not in space. This is not surprising: modern astronomy (especially since the formulation of Newton mechanics) was the first to open the scientific consideration of planet Earth as a nonunique object, the only known representative of a class, possibly large, of lifesustaining planets. ${ }^{2}$

Fantastic literature usually goes before speculative science. Thus, the idea that the universe is repetitive, in both spatial and temporal senses, that the same situations re-

* This paper has been partially sponsored by the Spanish Government, Secretaría de Estado de Investigación, Proyecto Ramón y Cajal RYC-2009-04042.

${ }^{1}$ Stoic philosophers maintained this position, apparently taken from Heraclitus, but it possibly goes back as far as Anaximander.

2 This idea was proposed long before as a philosophical speculation, without being widely accepted. Among those who held it, we can mention, for instance, Lucretius (De rerum natura, book II), cardinal Nicholas of Cusa and Giordano Bruno. The idea was also used in ancient science-fiction (e.g. Lucian of Samosata, True story, 2nd century AD). 
peat once and again, appears in stories such as La biblioteca de Babel, by Borges (1944), which describes a library, a parallel to the universe, which contains all the possible books, even those made of meaningless character strings. Every book contains exactly 1.312.000 characters. For every particular book, the library contains over thirty million almost identical copies which differ in just one character, which can be considered as copies with a single erratum; almost one quadrillion copies differing in two characters; and so forth. In addition, the library also contains the translation of every book to every language, either actual or just possible. However, both the library and the number of books in it are not infinite. If the library were actually infinite (Borges suggests this possibility at the end of the story) each book would be represented by an infinite number of copies.

Up to now, these ideas seemed proper only for mythology and fantastic literature, but recently a new possibility has arisen, that they can be derived from physical theories about the universe. Soon after the Big-Bang cosmology was established as the standard cosmological model, a few arguments were offered which, starting from apparently plausible assumptions, seem to bring us to the scenario of a universe with histories infinitely repeated in space.

The objectives of this paper are: (1) to summarize the two main arguments that end in this conclusion; (2) to discuss the controversial philosophical concept implicit in both: the actual possibility of an infinite universe; (3) and to criticize some possible errors in those two arguments.

To fulfil these goals, the remainder of this article is divided in this way: sections two and three describe the two most representative arguments that lead to a universe with an infinite number of histories repeated in space: the argument by Ellis and Brundrit, and the argument by Garriga and Vilenkin. Section four discusses the controversial nature of the thesis of the infinite universe, common to both arguments. Sections five and six point out other possible shortcomings in those arguments. Finally, section seven provides a few concluding comments.

\section{The Ellis-Brundrit (E-B) argument}

The argument proposed by George Ellis and G.B. Brundrit (see their 1979) starts from classic relativistic physics and the hypothesis that the universe is spatially infinite and homogeneous, and develops as follows:

1. The number of galaxies and planets in an infinite homogeneous universe is infinite.

2. The number of possible history lines in the configuration space of that universe is also infinite.

3. Among those history lines, a few could give room to the emergence of living beings based on DNA on the surface of a planet. The number of line histories compatible with life must be at least one, since we are here.

4. If the probability of DNA-based life should be greater than zero, an infinite universe will contain an infinite number of living beings of this type. To explain why this probability may be greater than zero, they claim the following: 
We assume that this is a stable property, that is, the probability remains finite [they mean nonzero] for a small range of conditions different from those holding in our own vicinity in the universe (e.g. a small change in average temperature or average matter-density would not reduce this probability to zero. (Ellis and Brundrit 1979, 38)

They mean by this that different but similar history lines would end up in the same or similar results.

5. The number of possible DNA-based living beings is finite, because the size of the DNA molecules cannot be arbitrarily large.

6. Therefore an infinite universe will contain an infinite number of copies of every possible living being. In a further step, and applying the same reasoning: since the possible material combinations making up a planet are also finite, we'll conclude that the universe must contain an infinity number of planets identical to Earth, including all the living beings on it. Ergo we live in a universe where events and histories are repeated in an infinity number of places. The authors explain it thus:

a. "...with an infinite number of histories to look at, it is difficult to provide an argument why a particular history should occur only once" (Ellis and Brundrit 1979, 38).

b. "If initial conditions are such that the probability of any event occurring is non-vanishing, then we expect that event to occur in infinitely many planetary systems" (Ellis and Brundrit 1979, 41, endnote b).

\section{The Garriga-Vilenkin $(G-V)$ argument}

In their paper "Many worlds in one" Joan Garriga and Alexander Vilenkin (Garriga and Vilenkin 2001) propose a different scenario, based on the decoherent histories (DH) interpretation of quantum mechanics (see Gell-Mann and Hartle 1990 and 1993). Their argument is developed in the following way:

1. They assume that the universe (or the inflationary multiverse, which seems to be their preferred option) is spatially infinite and homogeneous. That universe (or multiverse) can be divided into an infinity of causally disconnected regions (O-regions) defined by event horizons of the same size as ours.

2. They assume that space is quantic, so that every O-region can be divided in a finite number of cells.

3. In addition, they assume that this quantization is correctly described by the $\mathrm{DH}$ interpretation of quantum mechanics.

4. If the energy in every O-region is finite, since energy is quantized, it would be possible to divide its range of variation into a finite number of bins; therefore the number of possible histories in an O-region would be finite. In those environments, such as Earth, where planets and life can exist, energy can be considered finite, since we are far from gravity singularities. This means that gravity can be ruled out of this argument. 
5. Therefore, given that, by hypothesis, there is an infinite number of O-regions and the number of possible histories in each region is finite, every history must be repeated an infinite number of times in the universe.

It can be easily seen that the G-V and the E-B arguments are different in several respects:

- The E-B argument assumes a classical relativistic universe; the G-V argument starts from a quantum universe.

- The E-B argument states that the number of possible histories is infinite, and uses this assertion to prove its case. The G-V argument starts from the assertion that the number of possible histories is considered to be finite in every $\mathrm{O}$ region.

However, both share the postulate of the infinite universe, required in both scenarios. In sections 5 and 6 we shall criticize particular steps of the E-B and G-V arguments; but first, in section 4 , we will tackle the postulate of the infinite universe which, besides being common to both, is especially doubtful.

\section{The uncertain character of an infinite universe}

Both the E-B and the G-V arguments are based on the idea of a spatially infinite universe. However, if the history of physics can assess the initial credibility of a hypothesis, the postulate of a spatially infinite universe does not seem particularly promising. Paraphrasing Aristotle, we can say that nature abhors infinity..$^{3}$ Along the history of physics, once and again situations have emerged where infinities seemed impossible to avoid. Most of them have been abandoned and new improved theories have avoided the infinite. ${ }^{4}$

Just now we have two basic theories of physics, general relativity and quantum theory, which seem incompatible with one another. This makes it impossible to predict what happened before Planck's time, where both should be applied together. However, the two theories have a weak point: both predict infinities. Relativity, in the gravity singularities (the black holes and the Big Bang); quantum theory, in the energy of the vacuum and those quantities that must be renormalized in quantum field

\footnotetext{
${ }^{3}$ This idea is also spread among physicists. For instance, we can find it in Balbi: "We are used to thinking that nothing in nature is really infinite: but we seem oddly inclined to accept that the Universe might be. However, nature abhors infinity, and with good reason" $(2008,138)$. George Ellis himself supports this position in his famous review paper, where he relies on David Hilbert's analysis of the mathematical infinity as expressed in Hilbert's conclusion: "Our principal result is that the infinite is nowhere to be found in reality. It neither exists in nature nor provides a legitimate basis for rational thought $[. .$.$] The role that remains for the infinite to play is solely that of an idea [...] which transcends$ all experience and which completes the concrete as a totality [...]" (Hilbert 1964, 134-51; quoted in Ellis 2006, sect. 9.3.2).

${ }^{4}$ The historian of physics, Michael Riordan, for instance, has mentioned Dirac's opinion in a recent article about this point: "In the early 1980s, Nobel laureate Paul Dirac told Princeton University theorist Ed Witten that the most important challenge in physics was 'to get rid of infinity"' (Riordan 2012).
} 
theory. ${ }^{5}$ The experience provided by the history of physics seems to indicate that both theories could be simple approximations to a third more general theory, which would unify them and eliminate the infinities. Perhaps, when this theory is found, the cosmologic infinities may also disappear. In fact, one of the key objectives often stated in the pursuit of the theory that will overcome the limitations in the current pillars of theoretical physics, is the elimination of infinities. This is also used as a criterion to evaluate the various proposals currently being studied. For instance, in an article significantly entitled "Infinities as a measure of our ignorance", Francesca Vidotto summarizes with the following words the most common approach posed by physicists currently working in the field of quantum gravity:

Physics is about a quantitative description of the world around us. Infinities represent an end point for physics: their appearance means that we cannot associate a finite number to the system under study. Infinities provide the most tantalizing paradoxes to physicists: they point to the old assumptions that we have to give away in order to go beyond our present theories, toward the explorations of new level of energy, space and time. In this sense, every infinity and its overcoming is the turning point for a new paradigm. (Vidotto 2013, 10)

Such considerations are found in many other authors, ${ }^{6}$ though, of course, not all physicists share this attitude of total rejection of the existence of infinities in nature. ${ }^{7}$

However, while we wait for possible changes in our comprehension of the physical world, we must live with the usual claim, that the current standard cosmological model leaves open the possibility that the universe is infinite, since this is a possibility offered by Einstein's cosmological equation for $\mathrm{K} \leq 0$, where $\mathrm{K}$ represents, in that equation, the curvature of the universe.

${ }^{5} \mathrm{~A}$ recent description of the history of renormalization as a procedure to eliminate infinities in quantum field theory is (Close 2011).

${ }^{6}$ Thus, for instance, Carlo Rovelli has stated, in the context of his criticism to infinities in current Physics: "I think that what is truly infinite may just be the abyss of our ignorance" (Rovelli 2011, 174).

${ }^{7}$ Max Tegmark, for instance, even computes the distance to our nearest clone: "Is there another copy of you reading this article, deciding to put it aside without finishing this sentence while you are reading on? A person living on a planet called Earth, with misty mountains, fertile fields and sprawling cities, in a solar system with eight other planets. [...] You probably find this idea strange and implausible. [...] Yet it looks like we will just have to live with it, since the simplest and most popular cosmological model today predicts that this person actually exists in a Galaxy about $10^{10^{29}}$ meters from here. This does not even assume speculative modern physics, merely that space is infinite and rather uniformly filled with matter as indicated by recent astronomical observations" (Tegmark 2004, 459-91). This computation, however, does not take into account the difference in cardinality between the infinity of objects and the infinity of histories which we will discuss in the next section.

Some supporters of the possibility of an infinite universe with infinite repetitions, including Max Tegmark, now advocate one of the existing variants of the multiverse hypothesis (Tegmark 1998, 1-51). In the multiverse version proposed by Tegmark, the "mathematical multiverse," physical existence and mathematical consistency are one and the same, meaning that all mathematically consistent structures are physically existing universes. This amounts to postulating the existence of an infinite number of universes, which may contain as many infinities as may be defined in a consistent mathematical structure. However, Tegmark's ideas are taken with a grain of salt by the vast majority of physicists, while recent results point to the possibility that Tegmark's multiverse may not be consistent with the time independence of the natural laws in our universe (see Soler and Alfonseca 2013). 
It is important to realise that, even if we start from the standard cosmological model, the possibility that the universe is infinite can only be deduced if an additional postulate is accepted, namely the so-called 'cosmological principle', which asserts that the universe is homogeneous and isotropic at sufficiently large scales. This principle is necessary to extrapolate the cosmological description of the universe beyond the boundaries of the observable universe. However, its introduction gives rise to serious problems. ${ }^{8}$ In fact, the cosmological principle is being increasingly questioned from both sides: theoretical arguments and observational evidence. Therefore an agnostic position with respect to the extrapolation of the known universe beyond our horizon seems to be epistemologically reasonable, especially when that extrapolation leads to infinity. ${ }^{9}$

In consequence, in both the Ellis-Brundrit and Garriga-Vilenkin arguments, the mere fact that both depend on the assumption that the universe is infinite gives them a highly speculative character. However, for the sake of argument, let us assume that the universe can actually be infinite. In the next two sections we will try to show that, even so, the arguments described in the two previous sections cannot derive the conclusion that histories are repeated in space infinitely. Let us see why.

\section{A critic to the Ellis-Brundrit (E-B) scenario}

Now, let us discuss the E-B reasoning. To do this, we first consider the different steps in this reasoning and then, assuming that the premises (points 1 to 5) are right, mark that the conclusion (point 6) is partially wrong, for one of the ways in which the authors summarize it does not follow from the premises. Then we present two arguments that, in our view, invalidate the weakest link in the chain of premises.

1. Step 1 in the E-B argument states that an infinite homogeneous universe will contain an infinity of galaxies and planets. This is correct: if the universe is homogeneous, it will look the same everywhere. Since in our visible part of the

8 A comprehensive discussion of the problems raised by the justification of the 'cosmological principle' can be found in Beisbart (2009, 175-205). And with regard to empirical data, new structures of increasing size are found now and again. See, e.g. Clowes et al. (2012). Is there, in fact, a limit to the inhomogeneity of the universe? No one really knows. In his conclusion, Beisbart says: "The [Cosmological Principle (CP)] can be subjected to significant tests, and such tests have been carried out. But for the underdetermination problem, the most crucial question is: Can we extend the validity of the $\mathrm{CP}$ beyond the horizon on firm grounds? I have examined different strategies to justify the CP for the whole universe. Some of the strategies have been proposed in the literature, for instance that physical processes naturally drive the universe into a state in which the CP is obeyed. The result that I have obtained is that none of the strategies really works" (2009, 202). George Ellis also warns that the cosmological principle is not a part of the standard cosmological model, but an additional philosophical assumption: "The cosmological principle [...]: Just assume spatial homogeneity because it is the simplest case and you don't need anything more complex on the basis of current data. We simply adopt a philosophical principle as the basis of argument. This is essentially an a priori prescription for initial conditions for the universe [...] but it is not usually expressed that way" (Ellis 2006, section 4.2.2).

9 This is implicitly recognized by E-B when, at the beginning of their article, they assert that it "is based on philosophical rather than observational grounds [and therefore] should be treated with caution." 
universe we can see about $10^{11}$ galaxies, in an infinite universe their number must be infinite. As these entities are obviously countable, that infinity would be $\boldsymbol{\aleph}-0$ (the number of natural numbers).

2. On the other hand, the number of history lines mentioned in step 2 is not countable. In a classic universe with a continuous non-quantized space, which they are considering, that number would be the number of lines that could be drawn in the infinite volume of the configuration space ${ }^{10}$ defined by all the independent parameters of the universe. That infinity is greater than $\mathfrak{\aleph}-0$, and also greater than the continuum.

3. Step 3 is correct.

4. To estimate the probability of the emergence of living beings, according to step 4 , it would be necessary to estimate the number of history lines that make this emergence possible. Since the total number of lines is infinite (this is an E-B hypothesis), if that probability is to be greater than zero, the number of histories compatible with life must be infinite.

As E-B correctly indicate in an endnote, our existence does not imply that this probability is greater than zero. We must add that the same would happen, even if we discover a finite number of positive cases of life in other planets. To achieve an infinite number of histories, they assume that all the lines in a range in the configuration space around our history line (which did produce life) would give rise to the same or a very similar result. If the radius of that range were greater than zero, the number of history lines inside the range would be infinite.

5. For the time being, we accept step 5.

6. If all the previous steps were correct, most of step 6 (the E-B conclusion) would follow correctly from the premises. The 6.b quotation summarizes this conclusion. The 6.a quotation, however, is not valid. It should say just the opposite: ...with an infinite number of histories to look at, it is difficult to provide an argument why a particular history should occur more than once. This is so, because the infinity of lines is much larger than the infinity of living beings (which is $\mathfrak{\aleph}-0$, because living beings, like galaxies and planets, are always countable), therefore each planet compatible with life could be associated to a different history, even if their number were infinite.

We shall now discuss the premises. In our opinion, step 4 is unwarranted. Is it true that, by considering almost-identical histories, we get a probability greater than zero for the emergence of DNA-based living beings, as E-B affirm?

\footnotetext{
${ }^{10}$ Consider just the two parameters mentioned by E-B, temperature and matter-density. Both can take an infinite value (at the Big Bang and in the centre of a black hole, according to current theories). Therefore, their field of variation must be infinite. Ergo, the configuration space is infinite.
} 
This assertion can be refuted at least in two ways:

1. Refutation based on the probability that two beings/planets have very similar histories.

a. A history line in our universe (ours, for instance) at this point in time, is a curve in the configuration space of the universe (which is infinite by hypothesis) with a length of 13,700 million years (since the Big Bang to the present).

b. We shall call a history line g almost equal to another history line $\mathrm{h}$ when all the points in $g$ are inside a cylinder with a finite radius $\mathrm{E}$, built around the points of h.

c. Consider two planets, A and B. Let $\mathrm{h}$ be the history line of A. Which is the probability that $\mathrm{g}$ (the history line of $\mathrm{B}$ ) is almost equal to $\mathrm{h}$ ?

d. Although given $h$ there is an infinity of $g$ curves that comply with the definition, that probability is the quotient of the volume (in the configuration space) occupied by all the curves complying with the definition, divided by the volume occupied by all the curves. The former is the volume of a cylinder with radius $\mathrm{E}$ and a length of 13,700 million years, which is finite. The second is infinite (we have assumed that the configuration space is infinite). Ergo the probability that two planets $\mathrm{A}$ and $\mathrm{B}$ have almost equal curves is zero. This does not preclude that there may be planets supporting life apart from the Earth, but their number need not be infinite.

2. Refutation based on the chaotic character of the expansive universe.

The authors of the paper we are discussing suggest themselves a way to invalidate their result:

Now we do not necessarily believe that this final duplication actually occurs (arguments related to the uncertainty principle; free will; or the effects of very small environmental differences and the apparent continuity of the history of an individual, could provide a way out. (Ellis and Brundrit 1979, 38)

Taking their advice into account, an objection to their result could be raised as follows:

a. The uncertainty principle sets a minimum difference for the initial conditions of two different history lines.

b. The equations of classical relativistic physics (those used by E-B) are of the kind that give rise to chaotic behaviour.

c. Therefore two different history lines, which at the beginning will differ at least in the limits set by the uncertainty principle, must separate arbitrarily along their history, according to the definition of chaotic functions. This is even more true because the universe expands. And though the expansion of the universe seems to be un-chaotic (at least in the current state of our knowledge), it is wellknown that the behavior of galaxies and stars is chaotic. Since every history line of a living being must go through a galaxy, a star and a planet, it would be automatically affected by their chaotic behavior. 
d. Therefore there are no two almost equal history lines.

Would this argument be affected if the universe contained attractors where the history lines would converge? Those attractors could be:

a) Sinks (black holes). History lines passing through a black hole would be incompatible with life.

b) Cycles. They don't seem to exist in the universe. Perhaps they are incompatible with an expanding universe.

c) Saddles. They are unstable and sooner or later the history lines converging there would get apart in opposite directions.

d) Strange attractors. Those history lines attracted by them would get very near to the attractor, but not to one another. Given a point in one line, the next point would have an unpredictable position. Ergo two history lines attracted by the same strange attractor would be very different, even though superficially similar.

In summary, there are no two almost equal history lines, the probability of the emergence of living beings may be zero, and the Ellis-Brundrit argument cannot stand as formulated in their original paper. This result is important, as it gainsays the intuitive and well-known idea that in an infinite universe all the histories must be repeated automatically, including those of living beings on the Earth. ${ }^{11}$

We have thus shown three ways in which the E-B argument can be refuted:

1. The cardinality argument. The repetition of histories is avoided because the infinity of possible histories is larger than the infinity of objects (galaxies, planets, living beings) in an infinite universe.

2. The probability argument. To refute it, Ellis and Brundrit would need to prove that the number of histories leading to life is not concentrated in a finite volume in configuration space, or alternatively they would have to assert that the configuration space is finite. But then they would need to change their model of the universe, possibly going to a quantum model similar to that proposed by Garriga and Vilenkin.

3. The chaotic argument. Even if the probability argument could be refuted, the chaotic argument would make the existence of almost-equal history lines practically impossible.

11 As a sample of the usual association between the infinite universe and the repetition of histories, we can mention the following paragraph of a recent book: "In an infinite Universe, every possible event might happen. In fact, in an infinite Universe every possible event does happen. Not just that: it happens an infinite number of times. While I am writing these lines, somewhere else, in an infinitely remote and unreachable point of the Cosmos, well out of our horizon, an exact replica of me on a planet perfectly identical to ours is writing the exact same words, but with a spelling error. In some far-off place, another me is doing the same thing, but he wears a red sweater rather than a blue one" (Balbi 2008, 138). 


\section{A critic to the Garriga-Vilenkin $(G-V)$ scenario}

In our opinion the problem with this argument is that it relies on a set of questionable hypotheses. Therefore, their credibility is very low (because of the strong increase of uncertainty in a cascade of hypotheses). In fact, new unwarranted assumptions are needed at each step of the argument chain. Let us briefly sum up some of these assumptions, following the chain:

1. The very beginning of the argument, the hypothesis of an infinite space, has a speculative flavour, but let us forget about that now, since this is the basic hypothesis, which has been discussed in section 4 .

2. Space is assumed to be quantized, and quantum theory is applied to cosmology. This is just another conjecture. In fact, all the attempts in past decades to find an indication of the quantum character of space and gravity have been negative. ${ }^{12}$ Nonetheless, since many theorists suspect that the quantum formalism should be applicable to the description of gravity (and thus to the description of the universe as a whole), we can tentatively accept it.

3. Space quantization in the G-V argument is based on the DH interpretation of quantum mechanics. ${ }^{13}$ This interpretation has been the object of strong criticisms and is far from being the standard interpretation among specialists. ${ }^{14}$ From that point, the argument becomes very doubtful.

4. The authors themselves accept that the fourth step in their argument (the inference that the total number of distinct histories is finite) is valid only in a universe without gravity (i.e. empty). In our universe there are situations (inside black holes or at the Big Bang) where the energy density of a single region may be infinite, but they reject those situations because they are far from the world of human experience. The original text says the following (Garriga and Vilenkin 2001, 2-3):

In the absence of gravity, the boundedness of physical quantities usually follows from the finiteness of the energy of the system. The gravitational energy is not positive-definite, and in the presence of gravity the matter energy density can get arbitrarily large when it is compensated by equally large negative gravitational energy density. This happens, for example, during gravitational collapse in the interiors of black holes, in a recollapsing closed universe, and could happen in some extremely large quantum fluctuations. These extreme situations, however, are far removed from the low-energy world of the human experience, and it seems reasonable to isolate them when comparing the histories of different O-regions.

${ }^{12}$ For instance, it has been proposed that we could find an inkling of the quantum character of space by analyzing the arrival times of photons coming from remote cosmic explosions such as GRB [gamma ray bursts] and other high energy cosmic phenomena, studied by particle astrophysics. However, all the data currently available are consistent with the classic character of space. This is explained in more depth at Soler (2012).

${ }^{13}$ The unwarranted character of assumptions 2 and 3 in the G-V argument has also been emphasized by Henrik Zinkernagel (see his 2011, 215-41).

${ }^{14}$ An extensive critique of this interpretation can be found in (Dowker and Kent 1996). 
However, this reasoning presents several problems:

a) Obviously, for the time being at least, human beings do not have much interaction with black holes. It is an undue assumption that all other intelligent beings, or we ourselves in the future, will be in the same situation. Perhaps one day we'll be able to take advantage of black holes to reach relativistic speeds, in a similar way as when the Voyager spacecrafts used Jupiter's gravity to accelerate themselves toward Saturn. In that case, we'd be extracting energy from the black hole, which would become a part of our history.

b) But there is an additional important point that has not been considered in the G-V argument: the fact that all history lines did once go through a situation similar to the centre of a black hole, namely the Big Bang. Ergo the conditions with infinite energy density affect all the possible history lines, whose number could thus be infinite. As a result, the history lines would not have to repeat themselves, even in an infinite universe.

c) On the other hand, the idea that the histories of habitable regions can be described ignoring gravitational singularities does not take into account that these singularities exist inside the O-regions and clearly influence their dynamics. For instance, our planet rotates around the black hole in the centre of the Milky Way. Therefore, our own history depends to a certain extent on phenomena and regions related to a gravitational singularity. In summary, ignoring gravity while describing the O-regions does not seem reasonable.

So, even if we were to accept the (unproved) quantum cosmology and the (dubious) $\mathrm{DH}$ interpretation of quantum mechanics, we will not achieve the goal of the argument unless we accept another highly doubtful assertion, namely, that singularities can be disregarded.

Leaving aside the cascade of unwarranted hypotheses in their argument chain, there are other factors that $\mathrm{G}-\mathrm{V}$ have not taken into account, despite the fact that they can invalidate the outcome of their argument. For example, the fact that the universe expands may drastically affect their argument. In a stationary universe, a finite number of histories could have no option but repeat. In a dynamic universe, however, the number of bins in each O-region may increase with time in such a way that histories get apart from one another indefinitely. This would increase the number of possible histories (perhaps without limit) thus preventing repetitions. The $\mathrm{G}-\mathrm{V}$ argument cannot work without a proof that the number of bins relevant to building the histories is bounded in time in an expanding universe. It is their responsibility to provide it. And this is so, regardless of whether the gravitational singularities are considered as a part of the picture. G-V have not given any reason to suspect the existence of such a boundary, nor even signalled this difficulty.

Every one of the ideas postulated and the argumentative options mentioned in this list is doubtful, and the G-V argument requires all of them to be correct. Therefore, the scenario described by these authors must be considered highly speculative, to say the least. 


\section{Closing remarks}

From the discussion of the Ellis-Brundrit and Garriga-Vilenkin arguments in the preceding sections, it follows that the scenario of a universe where history repeats in an infinity of worlds spread throughout an infinite space cannot be considered the consequence of current physics and cosmology. At most, one could say that the possibility of this scenario is not definitively ruled out by current physics, although it requires the assumption of a number of very risky hypotheses. If someone were to assert, on the basis of the arguments discussed here, that physics proves the infinite repetition of histories in space, or even makes it likely, we should then reply that such opinions are just imagination, although they may be presented with the terminology of modern cosmology. Unfortunately, in practice, some authors ${ }^{15}$ and popular writers usually present such speculations as though they were very probable, almost proved by science. Even technical articles contain statements expressed with surprising certainty. For instance, in "Many worlds in one" Garriga and Vilenkin state: "The argument below is far from being a rigorous proof of these statements, but we do believe that it makes them rather plausible" $(2001,1)$.

"Rather plausible"? This surprising certainty becomes temerity when we go from the technical articles to popularizing books. For instance, in a recent book of this character, with the same title as the article discussed here, Vilenkin states:

We arrive at the inevitable conclusion that every single history should be repeated an infinite number of times. [...] And any history that has a nonzero probability will happen-or has happened: in an infinite number of O-regions! (2006, 111-12)

We believe that the analysis presented in this article shows that conclusion to be anything but inevitable.

\section{REFERENCES}

Balbi, Amedeo. 2008. The Music of the Big Bang. The Cosmic Microwave Background and the New Cosmology. Berlin: Springer.

Beisbart, Claus. 2009. Can we justifiably assume the Cosmological Principle in order to break model underdetermination in cosmology? Journal for General Pbilosophy of Science 40: 175-205.

Borges, Jorge Luis. 1944. La biblioteca de Babel. In Ficciones. Buenos Aires: Sur.

Close, Frank. 2011. The Infinity Puz:le: Quantum Field Theory and the Hunt for an Orderly Universe. New York: Basic Books.

Clowes, Roger G. et al. 2012. A structure in the early universe at $z \sim 1.3$ that exceeds the homogeneity scale of the R-W concordance cosmology. Available at: http://arxiv.org/abs/1211.6256

Dowker, Fay and Adrian Kent. 1996. On the Consistent Histories Approach to Quantum Mechanics. Journal of Statistical Physics 82: 1575-1646.

Eliade, Mircea. 1954. The Myth of the Eternal Return: Cosmos and History Princeton: Princeton University Press.

Ellis, George F.R. and Geoff. B. Brundrit. 1979. Life in the Infinite Universe. Quarterly Journal of the Royal Astronomical Society 20: 37-41.

${ }^{15}$ We must exclude Ellis from the set of authors that act in this way. In fact, as indicated above, the article by Ellis and Brundrit analyzed here mentions several factors that would invalidate their argument, and state clear reservations about the validity of the scenario they are describing. 
Ellis, George F.R. 2006. Issues in the Philosophy of Cosmology. In Philosophy of Physics (Handbook of the Philosophy of Science), ed. Jeremy Butterfield and John Earman. Amsterdam: North Holland, 1183-1286. Garriga, Joan and Alexander Vilenkin. 2001. Many worlds in one. Physical Review D 64: 043511-1-5.

Gell-Mann, Murray and John Hartle. 1990. Quantum mechanics in the light of quantum cosmology. In Complexity, Entropy and the physics of information, SFI studies in the Sciences of Complexity, vol. VIII, ed. Wojciech H. Zurek, 425-58. Reading: Addison-Wesley.

Gell-Mann, Murray and John Hartle. 1993. Classical equations for quantum systems. Physical Review D 47 (8): 3345-3382

Hilbert, David. 1964. On the Infinite. In Philosophy of Mathematics, ed. Paul Benacerraf and Hilary Putnam, 139-41. Englewood Cliffs: Prentice Hall.

Riordan, Michael. 2012. Tackling Infinity. American Scientist book review of (Close 2011). Available at http://www.americanscientist.org/bookshelf/pub/tackling-infinity.

Rovelli, Carlo. 2011. Some considerations on infinity in physics. In Infinity: New Research Frontiers, eds. Michael Heller and W. Hugh Woodin. Cambridge: Cambridge University Press. 167-175.

Soler, Francisco José. 2012. Discovery or construction? Astroparticle physics and the search for physical reality. Frankfurt: Peter Lang.

Soler, Francisco José and Manuel Alfonseca. 2013. Fine tunning explained? Multiverses and cellular automata. Journal for General Philosophy of Science 44: 153-172.

Tegmark, Max. 1998. Is 'the Theory of Everything' merely the ultimate ensemble theory? Annals of Physics 270: 1-51.

Tegmark, Max. 2004. Parallel universes. In Science and Ultimate Reality: Quantum Theory, Cosmology and Complexity, ed. John D. Barrow, Paul C.W. Davies and Charles L. Harper, 459-91. Cambridge: Cambridge University Press.

Vidotto, Francesca. 2013. Infinities as a measure of our ignorance. Available at: http://arxiv.org/pdf/1305.2358.pdf

Vilenkin, Alexander. 2006. Many worlds in one. New York: Hill and Wang.

Zinkernagel, Henrik. 2011. Some trends in the Philosophy of Physics. Theoria 26: 215-41.

Francisco José Soler Gil studied Physics and Philosophy at the University of Granada (Spain). He is a Doctor in Philosophy from the University of Bremen (Germany) and has carried out research at the University of Bremen and the Technical University of Dortmund (Germany). He is currently a research staff member "Ramón y Cajal" at the University of Seville (Spain). He has published several books and articles on Philosophy of Physics.

Address: Dpto. Filosofía, Lógica y Filosofía de la Ciencia, Universidad de Sevilla, C/ Camilo José Cela, s/n. Sevilla, 41018, Spain. E-mail: soler@uni-bremen.de

Manuel Alfonseca is a doctor in Electronics Engineering and Computer Scientist at the Universidad Politecnica of Madrid. He was a full professor, and now a honorary professor at the Department of Computer Science of the Universidad Autonoma of Madrid, where he was director of the Higher Polytechnical School (2001-2004). Previously, he was Senior Technical Staff Member at the IBM Madrid Scientific Center, where he worked from 1972 to 1994 . He has published over 200 papers and books on computer languages, simulation, complex systems, graphics, artificial intelligence, object-orientation and theoretical computer science, as well as popular science and juvenile literature, with different awards in these fields.

Address: Escuela Politécnica Superior, Universidad Autónoma de Madrid, Francisco Tomás y Valiente 11, Campus de Cantoblanco, 28049 Madrid, Spain. E-mail: manuel.alfonseca@uam.es 